DRAFT VERSION OCTOBER 28, 2019

Typeset using LATEX twocolumn style in AASTeX63

\title{
Infrared spectroscopy of the recent outburst in V1047 Cen (Nova Centauri 2005)
}

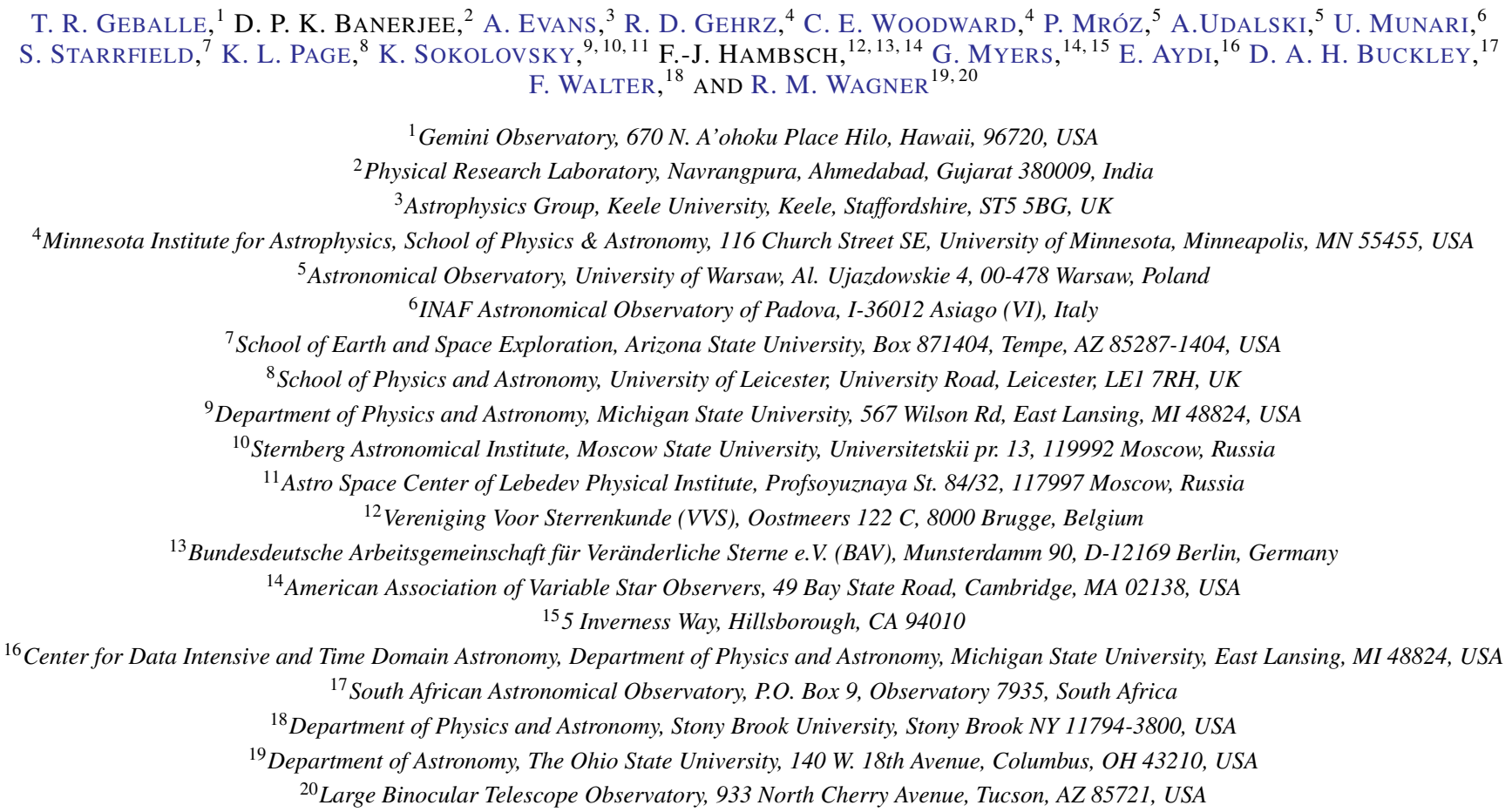

\section{ABSTRACT}

Fourteen years after its eruption as a classical nova (CN), V1047 Cen (Nova Cen 2005) began an unusual re-brightening in 2019 April. The amplitude of the brightening suggests that this is a dwarf nova (DN) eruption in a CN system. Very few $\mathrm{CNe}$ have had DN eruptions within decades of the main CN outburst. The 14 years separating the $\mathrm{CN}$ and DN eruptions of V1047 Cen is the shortest of all instances recorded thus far. Explaining this rapid succession of $\mathrm{CN}$ and DN outbursts in V1047 Cen may be challenging within the framework of standard theories for DN outbursts. Following a $\mathrm{CN}$ eruption, the mass accretion rate is believed to remain high $\left(\dot{M} \sim 10^{-8} \mathrm{M}_{\odot} \mathrm{yr}^{-1}\right)$ for a few centuries, due to the irradiation of the secondary star by the still-hot surface of the white dwarf. Thus a DN eruption is not expected to occur during this high mass accretion phase as DN outbursts, which result from thermal instabilities in the accretion disk, and arise during a regime of low mass accretion rate $\left(\dot{M} \sim 10^{-10} \mathrm{M}_{\odot} \mathrm{yr}^{-1}\right)$. Here we present near-infrared spectroscopy to show that the present outburst is most likely a DN eruption, and discuss the possible reasons for its early occurrence. Even if the present re-brightening is later shown to be due to a cause other than a DN outburst, the present study provides invaluable documentation of this unusual event.

Keywords: stars: individual (V1047 Cen) — novae, cataclysmic variables — infrared: stars

\section{INTRODUCTION}

A Cataclysmic Variable (CV) generally consists of a compact star (the primary) in a semi-detached binary system with a donor star that transfers matter onto the primary through the inner Lagrangian point via an accretion disk (AD). In classical and dwarf nova ( $\mathrm{CN}$ and $\mathrm{DN}$ respectively) systems the primary is a white dwarf (WD), and the secondary is a latetype star (see Warner 1995).

A $\mathrm{CN}$ eruption is the result of a thermonuclear runaway (TNR) in the degenerate material accreted on the surface of the WD (see Bode \& Evans 2012). Following the TNR, $\sim 10^{-6}-10^{-4} \mathrm{M}_{\odot}$ of material, enriched in $\mathrm{CNO}$, is ejected explosively at several $100-1000 \mathrm{~km} \mathrm{~s}^{-1}$. The system luminosity increases by a factor of as much as $\sim 15 \mathrm{mag}$. The bolometric 
luminosity remains approximately constant during the eruption, so the visual flux declines as the effective temperature of the stellar remnant increases. Once the ejecta become optically thin to $0.1-1.0 \mathrm{keV}$ photons, the source becomes a "super-soft X-ray source" (see Krautter 2012). CN eruptions are believed to repeat on time-scales of $\sim 10^{4}$ years.

Eruptions of DNe of the U Gem type are gentler affairs, and are due to an increase of the mass-flow through the AD as a consequence of a thermal instability within the AD itself; the amplitude of the outburst, which typically lasts a few days, is generally $\lesssim 5 \mathrm{mag}$, although WZ Sge stars can have outburst amplitudes up to $8 \mathrm{mag}$ (Warner 1995). There is little or no material ejected, and there is no enhancement of elemental abundances.

A small number of $\mathrm{CNe}$ have, since their eruptions, shown DN outbursts (Livio 1987; Mróz et al. 2016, and references therein), while some DNe seem to have circumstellar shells that appear to be the result of a previous $\mathrm{CN}$ outburst (e.g. Shara et al. 2007). In this cyclical scheme, CN-DN-CN (Shara et al. 1986), a DN eruption is not expected to follow within a decade of the $\mathrm{CN}$ outburst. This is because the cooling time-scale for the WD after the $\mathrm{CN}$ eruption is of the order of a century or more, during which high mass transfer rates from the secondary continue due to irradiation by the WD (Shara et al. 1986; Kovetz et al. 1988). Such circumstances are not conducive for a DN eruption unless special circumstances prevail (see Section 5 below). Hence the present outburst in V1047 Cen, if it is indeed a DN eruption, is a rare and exceptional event.

Here we present two epochs of near-infrared (NIR) spectroscopy of the CN V1047 Cen taken around the peak brightness of its 2019 outburst, and also present optical and NIR light curves that document the detection and evolution of the present re-brightening event.

\section{V1047 Cen (NOVA CENTAURI 2005)}

V1047 Cen (Nova Cen 2005) was discovered by Liller (2005) on 2005 September 1.031 at $m_{\text {vis }} \simeq 8.5$, but the 2005 eruption was not well observed. It was a CN of the "Fe II" spectroscopic type (Walter et al. 2012). The $\mathrm{H} \alpha$ line was found to have a FWHM of $\sim 840 \mathrm{~km} \mathrm{~s}^{-1}$ on day 3 (Liller 2005), and $\sim 1200 \mathrm{~km} \mathrm{~s}^{-1}$ on day 6.9 (Walter et al. 2012).

V1047 Cen (AT2019hik, Gaia19cfn) was found to be in outburst again by the Optical Gravitational Lensing Experiment (OGLE; Udalski et al. 2015) survey (Mróz \& Udalski 2019, see Fig. 1). The outburst started on 2019 April 6 and, at the time of writing (2019 mid-October, when it approached solar conjunction), had persisted for $\sim 160$ days. The nature of the slow rise to maximum, the outburst amplitude, and the subsequent variability, rule out a recurrent nova eruption for the 2019 event in V1047 Cen (see Anupama 2008). Currently, the duration of the 2019 April outburst is of the same

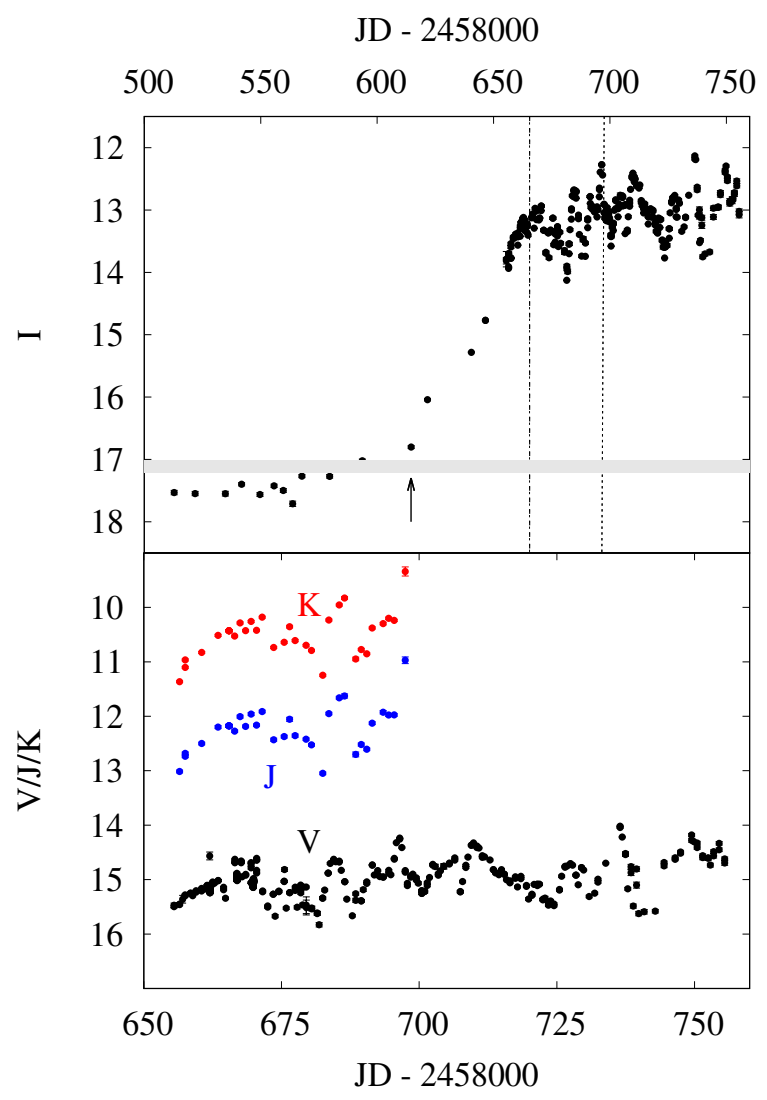

Figure 1. Top: I band light curve of V1047 Cen, showing the 2019 April outburst; data from the OGLE survey. Approximate time of the eruption is shown by the arrow. Horizontal shading denotes mean pre-2019 outburst $I$ magnitude and $\pm 1 \sigma$ variation from Mróz \& Udalski (2019). Times of the Gemini observations are indicated by the vertical dotted lines. Bottom: VJK light curve of V1047 Cen during eruption; note different JD scales. Data from the AAVSO and SMARTS (Walter et al. 2012) databases.

order as that of DN eruptions in the long period systems, and considerably longer than those in GK Per (e.g. Szkody et al. 1985; Salazar et al. 2017). The likelihood is that we are seeing a protracted DN eruption in a $\mathrm{CN}$ system.

High-resolution optical spectroscopy shortly after the 2019 April outburst was reported by Aydi et al. (2019a). At that time the spectrum was dominated by HI (FWZI > $2500 \mathrm{~km} \mathrm{~s}^{-1}$ ) and [O III] (FWZI $>1100 \mathrm{~km} \mathrm{~s}^{-1}$ ), which they suggest might have arisen in material ejected in the 2005 eruption. There were also narrow He I emission lines (FWZI $<300 \mathrm{~km} \mathrm{~s}^{-1}$ ), which may have originated in the 2019 DN eruption.

Timing analysis of the V1047 Cen light curve from the Transiting Exoplanet Survey Satellite (TESS; Ricker et al. 2014) indicated a possible $8.66 \pm 0.07$ hour periodicity (Aydi et al. 2019b), although this has yet to be verified. If this is confirmed to be the orbital period, it is at the higher end of $\mathrm{CV}$ orbital periods, and possibly suggests an evolved secondary. 
Table 1. Log of spectroscopic observations.

\begin{tabular}{lcrl}
\hline Date & $\begin{array}{c}\text { Grism, coverage } \\
\text { resolution }^{\mathrm{a}}\end{array}$ & $\begin{array}{r}\text { IT } \\
(\mathrm{s})\end{array}$ & Standard star \\
\hline 2019 Jun 30/Jul 1 & R3K, 1.9-2.5 $\mu \mathrm{m}$ & 1920 & HIP 63036 \\
2019 Jun 30/Jul 1 & $\mathrm{JH}, 0.9-1.8 \mu \mathrm{m}$ & 240 & HIP 67360 \\
2019 Aug 1/2 & R3K, 1.25-2.45 $\mu \mathrm{m}$ & 1260 & HIP 67360 \\
2019 Aug 1/2 & JH, 0.9-1.8 $\mu \mathrm{m}$ & 240 & HIP 67360 \\
\hline
\end{tabular}

a With the 0 "' 36 slit, for the $J H$ grism, $R$ ranges from $\sim 200$ near the short wavelength edge, to a maximum of $\sim 1100$ at $1.3-1.4 \mu \mathrm{m}$, dropping to $\sim 400$ near the long wavelength edge. For the R3K grism, $R$ varies from 1200 near $1.9 \mu \mathrm{m}$, increasing to 3200 near $2.2 \mu \mathrm{m}$, and then decreasing to $\sim 1200$ at $2.4 \mu \mathrm{m}$.

The distance and reddening to V1047 Cen are poorly constrained. The Gaia survey Data Release 2 (Bailer-Jones et al. 2018 , see this paper for the distance derivation, and its reliability) gives an estimated distance of $1.86 \mathrm{kpc}$, with lower and upper confidence bounds of $1.01 \mathrm{kpc}$ and $4.25 \mathrm{kpc}$ respectively. This renders it difficult to assign an accurate value for the reddening using $3 D$-extinction maps. For example, the extinction in the direction of V1047 Cen from Marshall et al. (2006) gives $E(B-V)$ values of $0.29,0.54$ and 2.54 at the Gaia lower, most probable, and upper distance estimates. Literature values for $E(B-V)$ give a wide range (e.g Senziani et al. 2008, give $E(B-V)$ in the range 1.28-1.38). Using the He II $\lambda 4686 \AA / \lambda 10124 \AA$ line ratio, $E(B-V)=1.1$ is suggested by Di Mille et al. (2019), who also used the $5780 \AA$ diffuse interstellar band to estimate $E(B-V)=1$.

In view of the considerable uncertainty in $E(B-V)$ we do not deredden the data here.

\section{OBSERVATIONS AND DATA REDUCTION}

Spectra of V1047Cen were obtained at the $8.1 \mathrm{~m}$ Gemini South Telescope using its facility spectrograph FLAMINGOS-2 (Eikenberry et al. 2004) in conditions of poor seeing and possible thin clouds. The log of the observations is given in Table 1. Data reduction was done using standard procedures for NIR data using IRAF ${ }^{1}$ and Figaro tasks. Because of the poor conditions under which the spectra were obtained, flux calibration of the spectra used broad band NIR photometry from SMARTS (see Fig. 1). The uncertainty in the flux scaling is $20 \%$ for the June 30/July 1 spectrum, and higher for the August 1/2 spectrum, as no NIR photometry was obtained on that night. However as we do not use absolute flux values in this study, our results and conclusions are not affected. The observed spectra are shown in Fig. 2.

\footnotetext{
${ }^{1}$ IRAF is distributed by the National Optical Astronomy Observatories, which is operated by the Association of Universities for Research in Astronomy, Inc. (AURA) under cooperative agreement with the National Science Foundation.
}

\section{THE INFRARED SPECTRA}

The IR spectra rule out the possibility that V1047 Cen is a stellar merger event (or "Luminous Red Nova"), as suggested by Mróz \& Udalski (2019). While stellar mergers have shown a similar slow rise to maximum, they also show a distinct shift to cooler spectral type, and display very specific NIR molecular features (e.g. AlO, $\mathrm{H}_{2} \mathrm{O}$ ) within 45 days of eruption. Our data show none of these characteristics. The absence of first overtone $\mathrm{CO}$ bands in the IR spectra (see Fig. 2) also rules out the possibility that V1047 Cen might be a symbiotic system.

Both spectra show lines of H I, He I and He II. The C, N and $\mathrm{O}$ lines that are generally seen in the NIR spectra of $\mathrm{RNe}$ - irrespective of whether the RN is of the T Pyx, T CrB or U Sco sub-types (Banerjee et al. 2009, 2010; Joshi et al. 2014) - are absent in V1047 Cen, with the exception of the O I line at $1.1289 \mu \mathrm{m}$ in August.

We have determined the velocities from the FWZIs of the $\mathrm{H} \mathrm{I} \mathrm{Pa}-\beta$ and $\mathrm{Br}-\gamma$ lines (Fig. 3) to be $\sim 2000 \mathrm{~km} \mathrm{~s}^{-1}$, as reported by Aydi et al. (2019a) for the optical H I lines, with no significant change between the two IR observations. The FWHMs of these lines are $\sim 1,500 \mathrm{~km} \mathrm{~s}^{-1}$, somewhat larger than the velocities reported by Liller (2005) and Walter et al. (2012) for $\mathrm{H}-\alpha$ during the 2005 eruption. The $\mathrm{Br}-\gamma$ line recorded at our highest resolution of $R \sim 3000$ clearly shows a double-peaked profile at one epoch, with a separation of $\sim 500 \mathrm{~km} \mathrm{~s}^{-1}$. This is consistent with the signature for emission lines originating from an AD (Horne \& Marsh 1986). Since the dip between the peaks is not very deep, a system at low inclination $\left(\lesssim 30^{\circ}\right)$ is indicated (Horne \& Marsh 1986) if the emission comes from the AD only.

Velocities of up to $2,000 \mathrm{~km} \mathrm{~s}^{-1}$ are also indicated by the He I $2.0587 \mu \mathrm{m}$ line, although there are dramatic changes in the line profile. (Based on the close agreements of the central wavelengths of the centers of the $\mathrm{HI}$ lines with their laboratory wavelengths, we conclude that the radial velocity of center of mass of the V1047 Cen system is no more than several tens of $\mathrm{km} \mathrm{s}^{-1}$ ). The July spectrum of this line displays a complex profile, with absorption extending from $\sim 0$ to $-2000 \mathrm{~km} \mathrm{~s}^{-1}$, sharp absorption troughs at -300 and $-1,400 \mathrm{~km} \mathrm{~s}^{-1}$, and weak emission extending from $\sim 0$ to at least $1600 \mathrm{~km} \mathrm{~s}^{-1}$. The troughs are replicated in the He I $\lambda 2.1138 \mu \mathrm{m}$ profile (see Fig. 3). In the August spectrum only a single absorption trough is present, at $-1,300 \mathrm{~km} \mathrm{~s}^{-1}$, the wing to higher negative velocities has weakened, and the emission now is much more extensive, from -800 to $+1600 \mathrm{~km} \mathrm{~s}^{-1}$.

The He I triplet at $1.0833 \mu \mathrm{m}$ is almost entirely in emission, but is more difficult to characterize quantitatively, due to the lower resolution at which it was observed and its blending on the long wavelength side with Pa- $\gamma(1.0941 \mu \mathrm{m})$. In the July 1 spectrum only a single weak P Cygni absorption is present at 


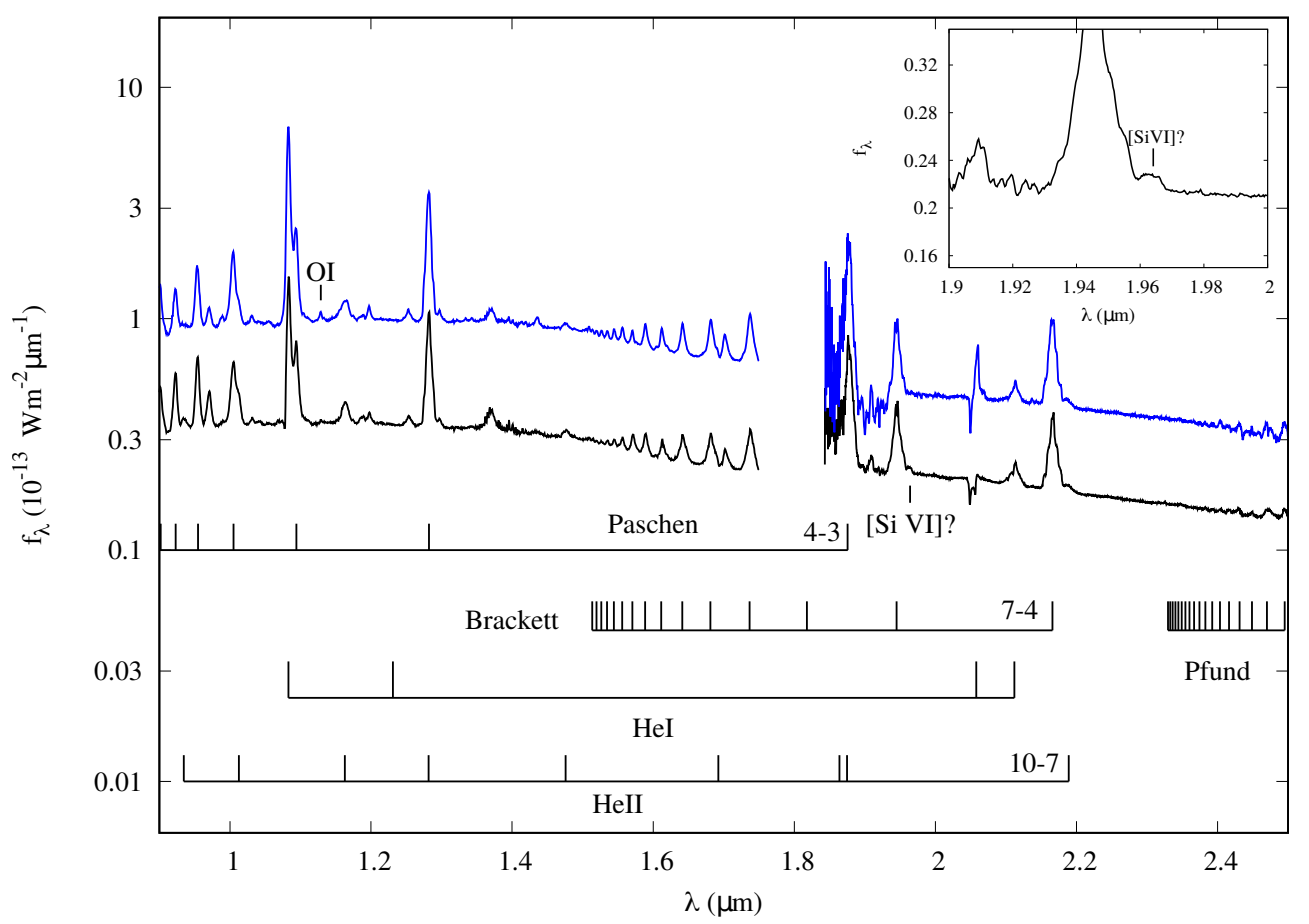

Figure 2. 0.9-2.5 $\mu \mathrm{m}$ spectra of V1047 Cen obtained on 2019 June 30/July 1 (black) and 2019 August 1/2 (blue). Hydrogen recombination and other lines are identified; for recombination lines, the transition for the longest wavelength member is shown. Inset shows the putative [Si VI] coronal line. The data behind this figure are available as a gzipped tar file.

$-1,600 \mathrm{~km} \mathrm{~s}^{-1}$, close in velocity to the more blueshifted absorption in the He I singlet profile, and with an absorption wing extending to $\sim-2,000 \mathrm{~km} \mathrm{~s}^{-1}$, similar to the singlet. In the August 2 spectrum absorption is completely absent, and the emission extends to $\sim-2,000 \mathrm{~km} \mathrm{~s}^{-1}$. At both times the FWHM of the line emission is $\sim 1,200 \mathrm{~km} \mathrm{~s}^{-1}$. In contrast, Aydi et al. (2019a) found relatively narrow (FWHM < $300 \mathrm{~km} \mathrm{~s}^{-1}$ ) He I emission lines in an optical spectrum obtained on July 19.86 UT. We note that the (deconvolved) FWHM of the O I line at $1.1289 \mu \mathrm{m}$, present in the August 2 spectrum, is $\sim 700 \mathrm{~km} \mathrm{~s}^{-1}$.

The cause of the P Cyg profile in the He I $2.0587 \mu \mathrm{m}$ line observed on July 1 is puzzling, but its reality is beyond doubt (see Fig. 3). We do not find a similar profile in the literature, though NIR spectra of DNe during outburst are rare (e.g. Howell et al. 2003). In quiescence such P Cyg profiles are not generally seen (see e.g. the DN sample observed by Dhillon et al. 2000). It is possible that the 2019 eruption has generated two separate outflows, as witnessed in the July 1 profile of the singlet line. However radiative transfer associated with this line is complex (see Geballe et al. 1984), and what we have observed may be a consequence of it rather than multiple outflows.

Many ultraviolet resonance lines (C IV 1549 A, Si IV $1397 \AA, \mathrm{N}$ V $1240 \AA$ ) in DNe often display P Cyg profiles, implying the presence of mass-loss in stellar winds (e.g. Holm et al. 1982; Warner 1995, and references therein). He II $1640 \AA$ and N IV $1719 \AA$ have also been found to have
P Cyg features, in RW Sex (Drew 1990), and it is possible

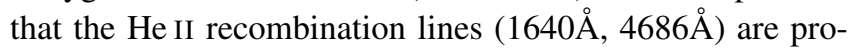
duced in both the disk and the wind (Warner 1995). We may thus speculate about the presence of a wind in this system which is responsible for the P Cyg profile in He I $2.0587 \mu \mathrm{m}$.

V1047 Cen also shows an intriguing $\mathrm{H} \alpha$ excess in images obtained prior to its current rebrightening. A SUPERCOSMOS (Hambly et al. 2001) H $\alpha$ image of the V1047 Cen field, obtained in 2001, shows no source at the position of the nova (see Fig. 4). On the other hand a VPHAS (Drew et al. 2014) image shows that V1047 Cen had a strong $\mathrm{H} \alpha$ excess in 2013, several years after its 2005 eruption, but before the 2019 event. The $\mathrm{H} \alpha$ excess in V1047 Cen is comparable with, or even stronger than, the excess in novae with giant secondaries and which show strong $\mathrm{H} \alpha$ in the SUPERCOSMOS images, as well as very strong $\mathrm{H} \alpha$ emission in their quiescent spectra (Anupama \& Mikołajewska 1999). The presence of an excess may lend support to our DN interpretation, since not only DNe in quiescence, but also old $\mathrm{CNe}$ and their remnants, show $\mathrm{H} \alpha$ emission in their spectra (Stauffer et al. 1979; Shafter \& Hessman 1988). We also note that N II

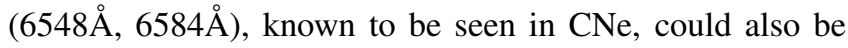
contributing partially to the $\mathrm{H} \alpha$ images. Nonetheless the strength of the $\mathrm{H} \alpha$ excess is puzzling and its evolution should be monitored.

There is some evidence for the presence of the [Si VI] $1.9641 \mu \mathrm{m}$ coronal line in the earlier spectrum (see Fig. 2). We note that a number of the $\mathrm{H}$ recombination lines have 


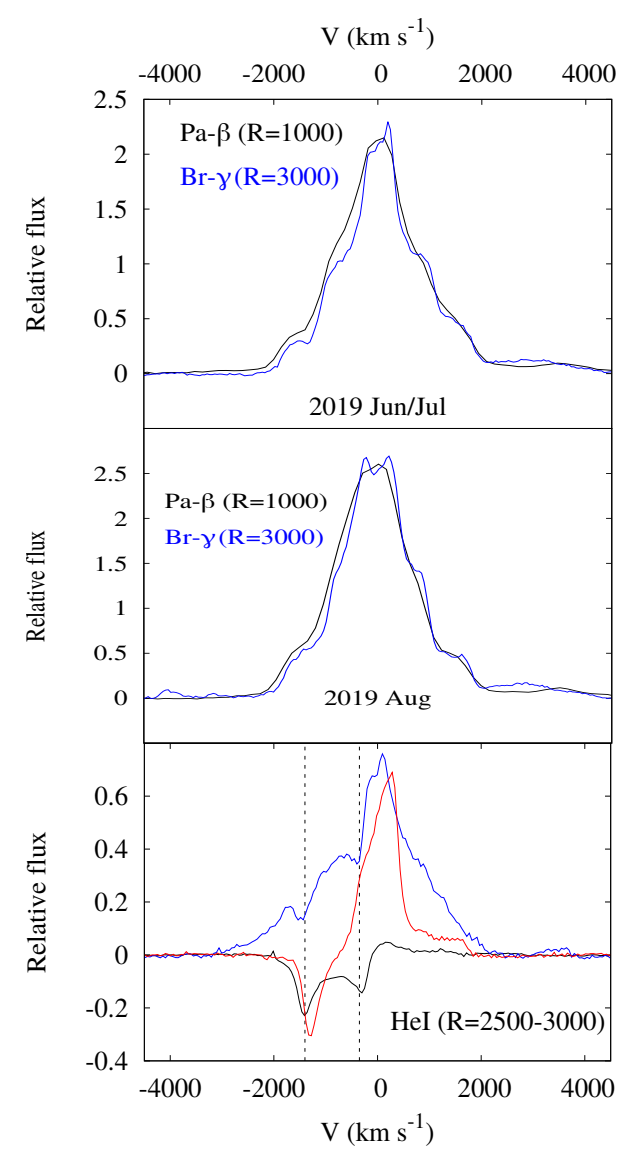

Figure 3. Top and middle: profiles of $\mathrm{Pa}-\beta$ and $\mathrm{Br}-\gamma$ lines, expressed as a function of velocity. Bottom: profiles of the He I $2.0587 \mu \mathrm{m}$ singlet for $2019 \mathrm{Jun} / \mathrm{Jul}$ (black) and Aug (red), and He I $2.1138 \mu \mathrm{m}$ for Jun/Jul (blue). Vertical lines denote troughs discussed in text. Numbers in brackets are spectral resolutions.

weak features on their red wings from He II but this is not the case for $\mathrm{Br}-\delta$. The ionization potential of $\mathrm{Si} \mathrm{V}$ is $167 \mathrm{eV}$, so the production of $\mathrm{Si}$ VI requires high energy conditions such as might occur in a shock. We have noted the likely presence of winds having different velocities; conditions for coronal emission might well occur in regions where the winds collide. We note that [Si VI] $1.9641 \mu \mathrm{m}$ emission has previously been detected in the CV TT Ari (Ramseyer et al. 1993).

\section{DISCUSSION}

If the 2019 April event in V1047 Cen is a DN eruption in a $\mathrm{CN}$ system, then we are witnessing a thermal instability in the AD. In their model of the disk-instability-induced DN eruptions in GK Per, Kim et al. (1992) found that AD temperatures in the range $6000-10000 \mathrm{~K}$ are expected. In order for the IR emission to reflect such temperatures, dereddening by $E(B-V) \gtrsim 1.6$ is required, for both the July and August spectra. This implies either that the reddening (and hence distance) to V1047 Cen is at the higher end of the range im-

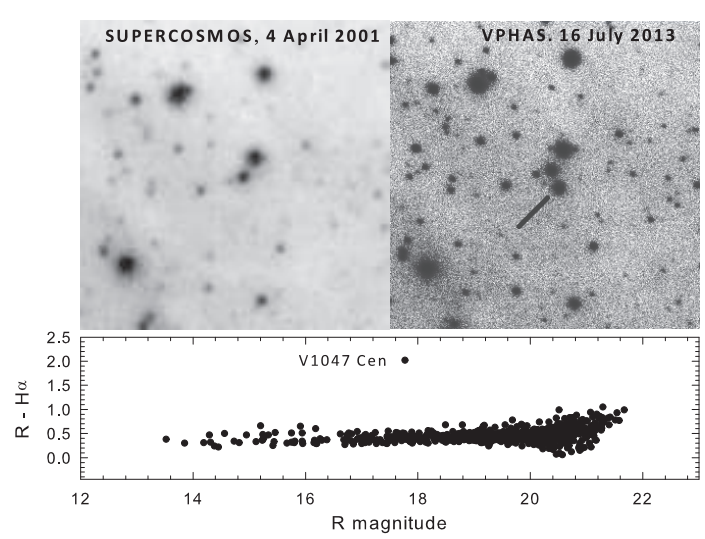

Figure 4. $75^{\prime \prime}$ (approx) square SUPERCOSMOS (top left) and VPHAS (top right) $\mathrm{H} \alpha$ images of the V1047 Cen field. North is up, east is right. The nova is arrowed on the VPHAS image. Bottom: $(R-\mathrm{H} \alpha)-R$ diagram for V1047 Cen field, showing large $\mathrm{H} \alpha$ excess in V1047 Cen.

plied by the Gaia data, or that what we are seeing is not a thermal instability event.

Only seven $\mathrm{CNe}$ are known to have exhibited DN outbursts within a few decades of their original $\mathrm{CN}$ outburst (see Vogt 1989; Honeycutt et al. 2011). The best documented DN outbursts are those of GK Per (CN 1901), V1017 Sgr (CN 1919) and V446 Her (CN 1960), while their subsequent DN eruptions were first recorded decades later: GK Per in 1948 (Bianchini et al. 1986), V446 Her (around 1991; Honeycutt et al. 2011) and V1017 Sgr (1973; Warner 1995). While DN outbursts have been observed regularly in GK Per and V446 Her (Bianchini et al. 1986; Honeycutt et al. 2011), only two DN outbursts are known to have occurred for V1017 Sgr, though it displayed a DN eruption in 1901 prior to its CN outburst (Salazar et al. 2017). The durations of individual DN events in these $\mathrm{CN}$ systems also vary substantially, lasting typically about 2 months for GK Per (Bianchini et al. 1986), 10-20 days for V446 Her (Honeycutt et al. 2011) and $\sim 200$ days for V1017 Sgr.

In this context the 2019 DN outburst in V1047 Cen has two notable characteristics. The duration of the outburst is relatively long, and as of mid-October 2019 the decline phase has yet to commence. Further, the DN event occurred only 14 years after the $\mathrm{CN}$ eruption, the shortest recorded gap between $\mathrm{CN}$ and DN eruptions. Explaining this behavior within the framework of the "hibernation" paradigm (Shara et al. 1986) may be challenging. In that theory for cyclic nova evolution, the mass accretion rate $\dot{M}$ remains high $\left(\dot{M} \sim 10^{-8} \mathrm{M}_{\odot} \mathrm{yr}^{-1}\right)$ on a time-scale of a few centuries after the $\mathrm{CN}$ eruption due to the irradiation of the secondary star by the still-hot WD (Shara et al. 1986; Kovetz et al. 1988). Eventually $\dot{M}$ declines, taking the AD through the DN phase and into hibernation, from which the $\mathrm{CV}$ reawakens after several millennia for the next $\mathrm{CN}$ eruption. The theory is sup- 
ported by the detection of ancient $\mathrm{CN}$ remnants around the DNe Z Cam (Shara et al. 2007), AT Cnc (Shara et al. 2012), and also by DNe outbursts preceding the main $\mathrm{CN}$ eruption of Nova Cen 2009 (Mróz et al. 2016). A DN eruption is not expected to occur during the high $\dot{M}$ phase that lasts for a few centuries after the $\mathrm{CN}$ outburst, as DN outbursts are believed to result from thermal instabilities associated with hydrogen ionization in the $\mathrm{AD}$ occurring during a regime of low mass accretion rate $\left(\dot{M} \sim 10^{-10} \mathrm{M}_{\odot} \mathrm{yr}^{-1}\right.$; Osaki 1974; Cannizzo 1993). Thus a special set of circumstances is needed to account for the early DN outburst in V1047 Cen. In the case of GK Per, DN eruptions are not surprising (Schreiber et al. 2000) as it has a large AD (Kim et al. 1992), consistent with its 2-day orbital period. Thus the critical mass transfer rate $\dot{M}_{\text {crit }}$ below which outbursts are possible is large (note that $\dot{M}_{\text {crit }}$ scales as $\left(R_{10}\right)^{2.6}$, where $R_{10}$ is the outer disk radius in units of $10^{10} \mathrm{~cm}$; Cannizzo 1993). Thus DN outbursts are possible in GK Per even at high accretion rates (Kim et al. 1992). It is possible that similar circumstances are responsible for the DN outburst of V1047 Cen but a robust determination of the orbital period is needed to verify this.

In summary, we have presented NIR spectra of the 2019 outburst of V1047 Cen. The presence of mostly H and He I lines in the spectra, the presence of double-peaked line profiles, and the nature of the light curve all suggest that a DN outburst has occurred. Such an event is rare, especially coming so soon after the $\mathrm{CN}$ outburst, and challenging to explain. The present and future outbursts of V1047 Cen could provide an opportunity to probe the $\mathrm{CN}$ to $\mathrm{DN}$ transition phenomenon and also test the generic mechanisms responsible for DN outbursts.

\section{ACKNOWLEDGMENTS}

This paper is largely based on observations obtained for program GS-2019A-DD-111 of the Gemini Observatory, which is operated by the Association of Universities for Research in Astronomy, Inc., under a cooperative agreement with the NSF on behalf of the Gemini partnership: the National Science Foundation (United States), National Research Council (Canada), CONICYT (Chile), Ministerio de Ciencia, Tecnología e Innovación Productiva (Argentina), Ministério da Ciência, Tecnologia e Inovação (Brazil), and Korea Astronomy and Space Science Institute (Republic of Korea).

The OGLE project has received funding from the National Science Centre, Poland, grant MAESTRO 2014/14/A/ST9/00121 to AU.

This work has made use of data from the European Space Agency (ESA) mission Gaia processed by the Gaia Data Processing and Analysis Consortium (DPAC), funding for which has been provided by national institutions, in particular the institutions participating in the Gaia Multilateral Agreement.

Based on data products from observations made with ESO Telescopes at the La Silla Paranal Observatory under programme ID 177.D-3023, as part of the VST Photometric $\mathrm{H} \alpha$ Survey of the Southern Galactic Plane and Bulge (VPHAS+, www.vphas.eu).

Nova research at Stony Brook is supported by a grant from the NSF. FMW acknowledges support from Stony Brook University for continued participation in the SMARTS consortium. DPKB is supported by a CSIR Emeritus Scientist grant-in-aid hosted by the Physical Research Laboratory, Ahmedabad. CEW acknowledges partial support from a USRA/NASA SOFIA contract. RDG is supported by NASA and the United States Air Force. KLP acknowledges support from the UK Space Agency. SS acknowledges support from a NASA Theory grant to ASU. DAHB thanks the National Research Foundation for research support.

We acknowledge with thanks the variable star observations from the AAVSO International Database contributed by observers worldwide and used in this research.

Facilities: Gemini South (FLAMINGOS-2), OGLE, Gaia, ESO, SMARTS, AAVSO

Software: IRAF (Tody 1986, 1993); Figaro (Shortridge et al. 2014)

\section{REFERENCES}

Anupama, G. C., Mikołajewska, J., 1999, A\&A, 344, 177

Anupama, G. C., 2008, in RS Ophiuchi (2006) and the Recurrent

Nova Phenomenon, eds A. Evans, M. F. Bode, T. J. O'Brien, M.

J. Darnley, ASP Conference Series, Vol. 401, p. 31.
Aydi, E., et al., 2019a, ATel 12885, 12975

Aydi, E., et al., 2019b, ATel 12889

Bailer-Jones, C. A. L., Rybizki, J., Fousneau, M., Mantelet,, G., Andrae, R., AJ, 156, 58 
Banerjee, D. P. K., et al., 2009, MNRAS, 399, 357

Banerjee, D. P. K., et al., 2010, MNRAS, 408, L71

Bianchini, A., Sabbadin, F., \& Dalmeri, I., 1986, A\&A, 160, 367

Bode, M. F., \& Evans, A., 2012, Classical Novae, Cambridge University Press, second edition

Cannizzo J. K., 1993, In: The Limit Cycle Instability in Dwarf Nova Accretion Disks. Advanced Series in Astrophysics and Cosmology 9, World Scientific, Singapore, p. 6

Cannizzo J. K., Shafter A. W., Wheeler J. C., 1988, ApJ, 333, 227

Cox, A. N., 2000, Allen's Astrophysical Quantities, Springer, New York, Berlin

Dhillon, V., et al., 2000, MNRAS, 314, 826

Di Mille, F., Congiu, E., Morrell, N., Boutsia, K., 2019, ATel 12893

Drew, J. E., 1990, in Physics of Classical Novae, IAU Colloquium 122, eds A. Cassatella \& R. Viotti, Springer Verlag, p. 228

Drew, J. E., et al., 2014, MNRAS, 440, 2036

Eikenberry, S., Elston, R., Raines, S. N., et al., 2004, SPIE, 5492, 1196

Geballe, T. R., Krisciunas, K., Lee, T.J., Gatley, I., Duncan, W.D., Garden, R., Becklin, E. E., 1984 ApJ 284, 118

Hambly, N. C., et al., 2001, MNRAS, 326, 1279

Holm, A. V., Panek, R. J., Schiffer, F. H., 1982, ApJ, 252, L35

Honeycutt, R. K., Robertson, J. W., Kafka, S., 2011, AJ, 141, 121

Honeycutt, R. K., Robertson, J. W., Turner, G. W., 1995, ApJ, 446, 838

Horne, K., \& Marsh, T. R., 1986, MNRAS, 218, 761

Howell, S., et al., 2003, A\&A, 399, 219

Joshi, V., et al., 2014, MNRAS, 443, 559

Kim, S. W., Wheeler, J. C., Mineshige, S., ApJ, 1992, 384, 269

Kovetz, A., Prialnik, D., Shara, M. M., 988, ApJ, 325, 828

Krautter, J., 2012, in Classical Novae, eds M. F. Bode \& A. Evans, Cambridge University Press, second edition, p. 232

Liller, W., 2005, IAUC 8596

Livio, M., 1987, Comments Astrophys., 12, 87

Marshall, D. J., Robin, A. C., Reylé, C., Schultheis, M., Picaud, S., 2006, A\&A, 453, 635
Mróz, P., et al., 2016, Nature, 537, 649

Mróz, P. \& Udalski, A., 2019, ATel 12876

Osaki, Y., 1974, PASJ, 26, 429

Ramseyer, T. F., Dinerstein, H. L., Lester, D. F., Provencal, J., 1993, AJ, 106, 1191

Ricker, G. R., et al., 2014, J. Ast. Tel. Inst. Systems, 1, 014003

Salazar, I. V., LeBleu, A., Schaefer, B. E., Landolt, A. U., Dvorak, S., 2017, MNRAS, 469, 4116

Schreiber, M. R., Gansicke, B. T., Cannizzo, J. K., 2000, A\&A, 362,268

Senziani, F, Skinner, G. K., Jean, P., Hernanz, M., 2008, A\&A, 485, 223

Shafter, A. W., \& Hessman, F. V., 1988, AJ, 95, 178

Shara, M. M., et al., 2007, Nature, 446, 159

Shara, M. M., et al., 2012, ApJ, 758, 121

Shara, M. M., Livio, M., Moffat, A. F. J., Orio, M., 1986, ApJ, 311, 163

Shortridge, K., Meyerdierks, H., Currie, M. J., Davenhall, C., Jenness, T., Clayton, M., 2014, Starlink Figaro: Starlink version of the Figaro data reduction software package, Astrophysics Source Code Library (ascl:1411.022)

Stauffer, J., Spinrad, H., Thorstensen, J., 1979, PASP, 91, 59.

Szkody, P., Mattei, J. A., Mateo, M., 1985, PASP, 97, 264

Tody, D., 1986, SPIE, 627, 733

Tody, D., 1993, in Astrononmical Data Analysis Software and Systems II, eds R. J. Hanisch, R. V. J. Brissenden, J. Barnes, ASP Conference Series, 52, 173

Udalski, A., Szymański, M. K., Szymański, G., 2015, Acta Astr., 65,1

Vogt, N., 1989, in Classical Novae, eds M.F. Bode and A.Evans, first edition, Wiley, p. 225

Walter, F. M., et al., 2012, PASP, 124, 1057

Warner, B., 1995, Cataclysmic Variable Stars, Cambridge University Press 